\title{
Norois
}

Environnement, aménagement, société

$203 \mid 2007 / 2$

Mobilité, hydrologie, loisir et tourisme, espace urbain

\section{Enjeux et impacts des barrages de Diama (Mauritanie) et Arzal (France) : des contextes socio- économiques et environnementaux différents pour de mêmes conséquences}

Stakes and effects of Diama (Mauritania) and Arzal (France) dams: different socio-economical and environmental context for same consequences

Aude Nuscia Taïbi, Mohamed el Habib Barry, Maxime Jolivel, Aziz Ballouche, Mohamed Lemine Ould Baba et Gérard Moguedet

\section{OpenEdition}

Journals

Édition électronique

URL : https://journals.openedition.org/norois/1536

DOI : $10.4000 /$ norois. 1536

ISBN : 978-2-7535-1551-2

ISSN : 1760-8546

Éditeur

Presses universitaires de Rennes

Édition imprimée

Date de publication : 1 juin 2007

Pagination : 51-66

ISBN : 978-2-7535-0511-7

ISSN : 0029-182X

Référence électronique

Aude Nuscia Taïbi, Mohamed el Habib Barry, Maxime Jolivel, Aziz Ballouche, Mohamed Lemine Ould Baba et Gérard Moguedet, «Enjeux et impacts des barrages de Diama (Mauritanie) et Arzal (France) des contextes socio-économiques et environnementaux différents pour de mêmes conséquences », Norois [En ligne], 203 | 2007/2, mis en ligne le 01 juin 2009, consulté le 13 janvier 2022. URL : http:// journals.openedition.org/norois/1536 ; DOI : https://doi.org/10.4000/norois.1536 


\title{
Enjeux eT impacts des barrages de Diama (Mauritanie)
}

et Arzal (France) : Des ConteXtes socio-ÉCONOMiques

\section{ET ENVIRONNEMENTAUX DIFFÉRENTS POUR DE MÊMES CONSÉQUENCES}

\author{
Aude Nuscia Taïbi ${ }^{1}$, Mohamed el Habib Barry ${ }^{2}$, Maxime Jolivel ${ }^{2}$, Aziz Ballouche ${ }^{2}$, \\ Mohamed Lemine Ould BabA ${ }^{3}$, Gérard Moguedet $^{3}$ \\ ${ }^{1}$ CARTA - ESO UMR 6590 CNRS \\ (Université d'Angers), \\ 35 rue de La Barre - 49000 ANGERS \\ ${ }^{2}$ Laboratoire Paysages et Biodiversité \\ (Université d'Angers), \\ 2 boulevard Lavoisier - 49000 ANGERS \\ ${ }^{3}$ UICN et Groupe de Recherche sur Les Zones Humides (GREZOH) \\ (Université de Nouakchott), \\ Faculté des Sciences et Techniques, BP 5026 - Nouaкchotт (Mauritanie) \\ nucia.taibi@univ-angers.fr,barrymohamed@yahoo.fr,maxime.jolivel@laposte.net, \\ mohamed.lemine.ould.baba@iucn.org, aziz.ballouche@univ-angers.fr, gerard.moguedet@univ-angers.fr
}

\section{RÉSUMÉ}

Les barrages d'Arzal sur la Vilaine (France) et de Diama sur le fleuve Sénégal à la frontière mauritano-sénégalaise, ont les mêmes fonctions principales, stratégiques pour les régions et pays où ils ont été bâtis, puisqu'ils permettent de constituer d'énormes réserves d'eaux douces en zones estuariennes, et ceci malgré des contextes environnementaux et socio-économiques très différents. S'ils ont favorisé l'émergence d'autres atouts, ils ont aussi eu des conséquences importantes sur les écosystèmes et les activités traditionnelles locales et régionales.

Les actions de remédiation mises en ouvre tardivement, et qui auraient sans doute pu être plus ambitieuses, ont eu des résultats mitigés dont les effets induits laissent les populations et les écosystèmes dans une situation de fragilité et de vulnérabilité face à toute crise environnementale (sécheresse, crue/inondation, etc.), socio-économique ou encore politique, comme on a pu le constater par exemple lorsque les relations mauritano-sénégalaises se sont dégradées.

MotS CLÉS : Arzal - Barrages estuariens - Diama - Fleuve Sénégal - Impacts environnementaux et socio-économiques - Remédiation - Vilaine.

\section{ABSTRACT}

Stakes and effects of Diama (Mauritania) and Arzal (France) dams: different socio-economical and environmental context for same consequences

The similar dams of Arzal (Vilaine, France) and Diama (Mauritania-Senegal frontier) have same strategic functions for the regions and countries where they are built as fresh waters storage. They also had similar consequences over the ecosystems and local and regional activities. 
The imperfect results of the belated remediations also had as disastrous induced effects and leave the populations and ecosystems in a fragile and vulnerable situation if confronted with any environmental (drought, flood...), socio-economical or political (relations between Mauritania and Senegal) crisis.

KEY WORDS : Arzal - Diama - Environmental and socio-economical impacts - Estuarine dams - Remediation - Senegal river - Vilaine river.

Les barrages estuariens d'Arzal sur la Vilaine en France (fig. 1) et de Diama sur le fleuve Sénégal à la frontière mauritano-sénégalaise (fig. 2), ont été construits sur un modèle très semblable et avec des objectifs similaires malgré des contextes environnementaux et socio-économiques très différents. Le premier est en effet construit en climat tempéré océanique à l'exutoire d'un bassinversant de $10000 \mathrm{~km}^{2}$, alors que le second est situé en milieu sahélien, sur l'estuaire d'un fleuve dont le bassin-versant couvre une superficie d'environ $340000 \mathrm{~km}^{2}$.

Malgré ces différences importantes, ces ouvrages aux fonctions principales identiques, ont généré d'importantes conséquences sur les écosystèmes et les activités traditionnelles pratiquées sur ces espaces humides aux caractéristiques remarquables.

Si les contextes de mise en place et de gestion diffèrent ainsi que les actions mises en œuvre pour réduire leurs impacts et notamment restaurer les milieux et les activités économiques liées, ces mesures compensatoires auraient certainement pu être, dans les deux cas, plus ambitieuses. La présentation des mesures mises en œuvre pour remédier aux conséquences du barrage de Diama et de la réalité de leur impact par télédétection notamment, reprend des données publiées précédemment par les auteurs (Taïbi et al., 2006).

Ces exemples illustrent parfaitement que ce sont d'abord les contraintes économiques qui comptent pour les décideurs, ce qui peut se comprendre aisément lorsque ces équipements sont stratégiques. Les conséquences environnementales de ces aménagements ne constituent pas en revanche des préoccupations prioritaires, aussi bien en Europe qu'en Afrique. La mise en oeuvre partielle ou tardive des mesures compensatoires pour réduire ces impacts nuit beaucoup, surtout dans le contexte actuel, à l’image positive apportée par ces aménagements.

\section{Les fonctions et les contextes de la mise en place des deux barrages}

Arzal construit en 1970 sur l'estuaire de la Vilaine (fig. 1) et Diama, sa copie conforme sur le plan technique, en 1986 sur l'estuaire du fleuve Sénégal (fig. 2), sont deux barrages poids en
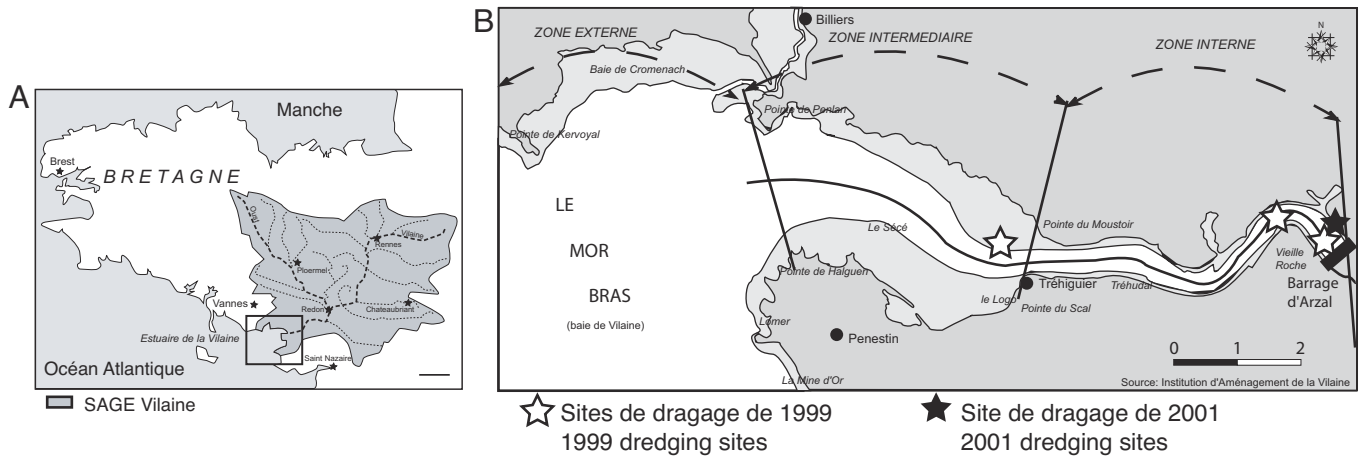

Figure 1 : Bassin versant et estuaire de la Vilaine et localisation du barrage d'Arzal Vilaine river catchement area and estuary. Arzal dam location 


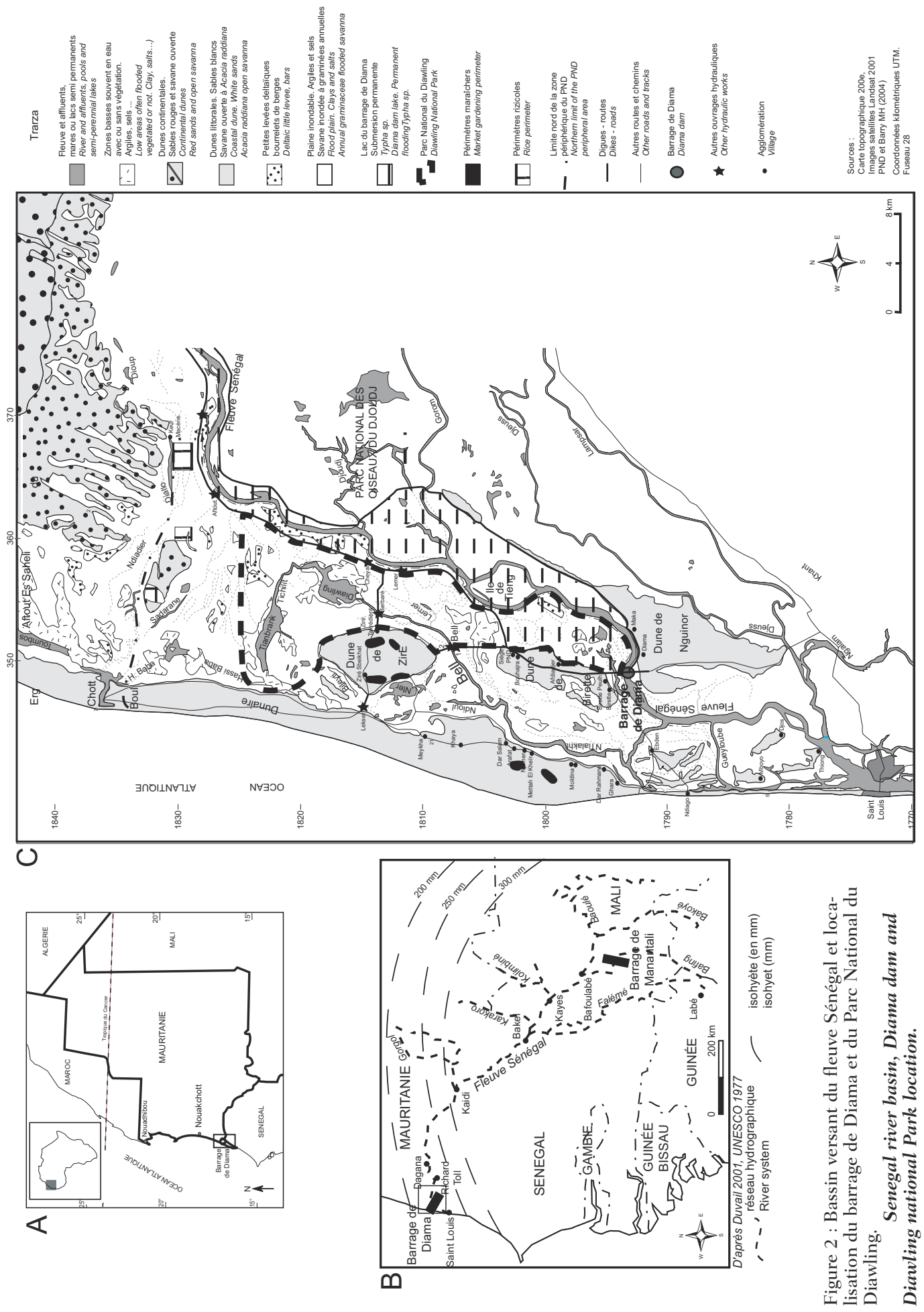


terre, c'est à dire constitués de remblais en enrochements et qui présentent des caractéristiques techniques très proches (tableau 1).

Diama et Arzal sont deux ouvrages dont la fonction principale est la même : ce sont deux barrages anti-sel destinés à créer des réserves d'eau douce en empêchant la remontée des eaux salées marines vers l'amont.

Ils s'ouvrent en période de crue pour assurer l'écoulement normal du fleuve et se ferment en période d'étiage pour empêcher la remontée de la langue salée, constituant ainsi une réserve d'eau douce.

Paradoxalement à ces similitudes techniques, les contextes physiques locaux et régionaux de mise en place de ces ouvrages, varient beaucoup d'un site à l'autre (tableau 2).

\begin{tabular}{|l|l|l|}
\cline { 2 - 3 } \multicolumn{1}{l|}{} & Arzal & Diama \\
\hline Type Barrage & Barrage poids en terre et ouvrages en béton & Barrage poids en terre et ouvrages en béton \\
\hline Reserve eau douce & $50000000 \mathrm{~m}^{3}$ & $\begin{array}{l}50 \text { millions } \mathrm{m}^{3} \text { à la cote }+1,50 \mathrm{~m} \text { IGN mais } \\
\text { conçu pour retenir } 585 \mathrm{millions} \mathrm{m}^{3} \text { à la cote } \\
2,50 \mathrm{~m} \text { IGN }\end{array}$ \\
\hline $\begin{array}{l}\text { Digue } \\
\begin{array}{l}\text { Enrochement et terre } \\
370 \mathrm{~m} \text { de long } \\
20 \mathrm{~m} \text { de large en crête à la côte }+4,50 \mathrm{~m} \text { NGF }\end{array}\end{array}$ & $\begin{array}{l}\text { Enrochement, sable-argile } \\
400 \mathrm{~m} \text { de long } \\
\text { + « digue de bouchure » de } 440 \mathrm{~m} \text { de long } \\
\text { obturant le lit mineur } \\
2 \text { « digues de fermeture » du lit majeur }(2500 \mathrm{~m} \\
\text { de long en rive droite et } 440 \mathrm{~m} \text { en rive gauche }\end{array}$ \\
\hline $\begin{array}{l}\text { Écluse de navi- } \\
\text { gation }\end{array}$ & $\begin{array}{l}13 \mathrm{~m} \text { de large } \mathrm{x} 85 \mathrm{~m} \text { de long } \\
\text { Passage caboteurs de } 1500 \mathrm{t}\end{array}$ & $\begin{array}{l}13 \mathrm{~m} \text { de large } \mathrm{x} 175 \mathrm{~m} \text { de long } \\
\text { Passage caboteurs de } 1500 \mathrm{t}\end{array}$ \\
\hline $\begin{array}{l}\text { Évacuateur de } \\
\text { crue }\end{array}$ & $\begin{array}{l}5 \text { passes de } 18 \mathrm{~m} \text { de large avec vannes segments } \\
\text { relevables }\end{array}$ & $\begin{array}{l}7 \text { passes de } 20 \mathrm{~m} \text { de large avec vannes segments } \\
\text { relevables } \\
170 \mathrm{~m} \text { long } \mathrm{x} 35 \mathrm{~m} \text { large }\end{array}$ \\
\hline
\end{tabular}

Tableau 1 : Caractéristiques techniques des deux barrages (Source : Arzal : Maillocheau 1980, IAV 1995; Diama : BDPA/SCETAGRI 1994)

Technical caracteristics of both dams

\begin{tabular}{|l|l|l|}
\cline { 2 - 3 } \multicolumn{1}{c|}{} & Arzal (Vilaine) & Diama (fleuve Sénégal) \\
\hline Climat & Tempéré océanique & Sahélien \\
\hline Précipitations moyennes annuelles & Entre 650 et $800 \mathrm{~mm}$ & Entre 200 et $500 \mathrm{~mm}$ \\
\hline Bassin-versant drainé & $10882 \mathrm{~km}^{2}$ & $337000 \mathrm{~km}^{2}$ \\
\hline Débit moyen & $80 \mathrm{~m}^{3} \cdot \mathrm{s}^{-1}$ & $640 \mathrm{~m}^{3} \cdot \mathrm{s}^{-1}$ \\
\hline Régime & Pluvial océanique & Pluvial tropical \\
\hline Longueur rivière & $225 \mathrm{~km}$ & $1790 \mathrm{~km}$ \\
\hline
\end{tabular}

Tableau 2 : Contexte physique des deux barrages

$$
\text { Physical context of both dams }
$$

Une autre distinction réside dans le fait que la gestion du barrage de Diama, assurée par l'OMVS (Organisation pour la Mise en Valeur du fleuve Sénégal) organisme inter-états dont le siège est à Saint-Louis au Sénégal, est étroitement liée à celle du barrage-réservoir de Manantali achevé en 1988 très en amont du fleuve Sénégal (fig. 2b), et dépend beaucoup de ses lâchers, fonctions des objectifs de soutien du débit d'étiage et de production d'hydroélectricité.

La retenue de Diama alimente en eau des périmètres irrigués, en particulier rizicoles, mais alimente aussi en eau potable les villes de Saint-Louis et Dakar au Sénégal et probablement Nouakchott en Mauritanie dans le futur. 
La retenue d'Arzal, administrée par l'IAV (Institut d'Aménagement du bassin de la Vilaine), et qui gère également l'ensemble du bassin-versant de la Vilaine, avec une réserve d'eau douce de 50 millions de $\mathrm{m}^{3}$ couplée à une usine de production d'eau potable, alimente en eau potable la presqu'île guérandaise (La Baule, Le Croisic) qui reçoit un afflux de population en période estivale, mais aussi la ville de Saint-Nazaire et doit alimenter prochainement la région de Vannes également très touristique (golfe du Morbihan) et même, à terme, les régions de Redon et de Rennes, soit environ 1,5 million de personnes. C'est le plus grand barrage estuarien français destiné à stocker de l'eau douce brute potabilisable.

Ces barrages ont également d'autres fonctions puisqu'ils permettent le franchissement des estuaires où ils sont construits. Diama est le seul point de franchissement routier dans la région aval du Fleuve Sénégal qui sert de frontière naturelle entre la Mauritanie et le Sénégal et permet de relier Nouakchott, et toute la Mauritanie, à Saint-Louis au Sénégal. C'est donc un aménagement essentiel permettant les échanges économiques entre les deux pays riverains du Fleuve.

L'ouvrage d'Arzal permet également le franchissement de l'estuaire de la Vilaine et devait être un des maillons essentiels de ce que l'on appelait alors la Route Bleue permettant de relier la Bretagne au sud-ouest de la France, en passant également par le pont de Saint-Nazaire sur la Loire. Malheureusement en raison de problème de stabilité de l'ouvrage, cet axe qui devait être structurant, n'a pu servir aux poids lourds et le pont est surtout emprunté par les véhicules légers. Le grand projet de Route bleue a été ainsi plus ou moins abandonné et pour gagner le sud-ouest depuis la Bretagne, Nantes et le pont de Cheviré sont un passage quasi obligé.

Les barrages sont également censés limiter les inondations, à l'aval pour le fleuve Sénégal, et en particulier à Saint-Louis, à l'amont pour la Vilaine et en particulier la région de Redon en gérant l'écoulement des eaux issues du bassin-versant (niveau le plus bas possible en période de crue à $+0,5 \mathrm{~m} \mathrm{NGF}$ ). La pente naturelle très faible dans la partie aval de ces deux fleuves favorisait, avant la construction des ouvrages, la remontée de la marée dynamique sur de longues distances vers l'amont. Les eaux salées ne remontent plus aujourd'hui au-delà de $12 \mathrm{~km}$ contre $40 \mathrm{~km}$ avant barrage dans la Vilaine et ne concerne plus que l'aval de Diama contre $200 \mathrm{~km}$ avant barrage dans le fleuve Sénégal.

Ceci étant, ces ouvrages n’ont pas empêché des inondations catastrophiques en octobre 2003 à Saint-Louis et en 1995 et 2000 dans la région de Redon.

Le barrage d'Arzal a par ailleurs dynamisé l'activité de plaisance, de l'estuaire de la Vilaine jusqu’à Redon. On compte ainsi 15000 passages annuels de bateaux par l'écluse d'Arzal et 2500 bateaux en mouillage en amont du barrage. Le développement de la navigation qui était également prévu sur le fleuve Sénégal avec la création du barrage de Manantali, n’en est qu’à un stade embryonnaire.

Arzal comme Diama ont permis également le développement des activités agricoles. L'assèchement des marais en aval de Redon, milieux particulièrement riches, a ainsi permis aujourd'hui leur exploitation en prairies pour l'élevage ou en grande culture notamment de céréales. Dans la vallée du fleuve Sénégal c'est la riziculture sur périmètres irrigués qui a été développée et on note également la multiplication des périmètres maraîchers sur les dunes en bordure de la réserve de Diama.

\section{Une dégradation majeure des écosystèmes et des activités}

Ces barrages ont généré dans un deuxième temps de profondes mutations et dégradations environnementales et socio-économiques qui sont venues rapidement tempérer voire annuler les effets positifs recherchés de ces fonctions initiales.

\section{Les impacts du barrage de Diama dans le bas delta du fleuve SÉnÉgal}

Dans le bas delta du fleuve Sénégal, ces conséquences ont été rapidement mises en évidence par des travaux postérieurs aux barrages comme ceux de P. Michel et al., dès 1993 ou S. Duvail en 2001 
Aude Nuscia TAÏBI ET AL.

puis en collaboration avec O. Hamerlynck en 2003 par exemple. Ces observations ont été complétées depuis par M. Barry (2004) ainsi que par télédétection satellitaire par A. Taïbi et al. (2006).

Avec l'endiguement des deux rives sur plus de 100 km (fig. 2c), le phénomène inondation-exondation des plaines inondables n'existe plus et on constate une modification profonde des milieux. L'inondation partielle étant artificialisée et liée à l'ouverture temporaire d'ouvrages, les cycles de la végétation sont désormais désaisonnalisés et s'adaptent aux arrivées d'eaux.

Par ailleurs, les terres du Bas Delta ont été soumises à des phénomènes de salinisation importants depuis la construction du barrage. Ces processus mis en évidence par télédétection satellitaire dans des travaux antérieurs des auteurs (Taïbi et al., 2006) sont nettement discriminés par des classifications diachroniques des 3 premières composantes d'analyses en composante principale réalisées sur des images satellites masquées de 1992 et 2001 (fig. 3), particulièrement au niveau de l'estuaire à l'aval de Diama encore soumis au cycle de la marée, ainsi que dans le bras du N'Tiallakh. L'absence de recharge de la nappe superficielle par les eaux douces dans l'ancienne plaine d'inondation favorise la remontée d'un biseau salé et les phénomènes d'ascension capillaire des eaux salées superficielles. Ainsi au nord, les anciennes cuvettes de la plaine inondable, peu ou pas atteintes par les inondations de Diama, ont eu tendance à évoluer en sebkhas. Cette salinisation est accentuée aux environs de la retenue en raison de la pression hydrostatique qu'elle provoque (Hamerlynck et Duvail, 2003). La combinaison de la sécheresse, de la salinisation des terres et des eaux, de la disparition des crues et de l'inondation prolongée en amont du barrage, ont considérablement appauvri la flore de la plaine alluviale, certaines espèces ayant totalement disparu. La mangrove s'est ainsi fortement réduite partout, et souvent, lorsqu'elle subsiste, les peuplements d'Avicennia africana ont supplantés Rhizophora racemosa. Les superficies occupées par Sporobolus robustus, exploitées artisanalement par les populations locales, ont également fortement diminué (Duvail, 2001).

Les formations de savane arborée ont également souffert, en combinaison avec une coupe abusive des arbres. Elles sont réduites souvent à quelques individus (Acacia, Balanites et Adansonia) en bordure des plaines inondables ou sur les massifs dunaires de Ziré, Birette et Ebden et la dune côtière, qui sont eux en nette revivification comme le soulignent les indices de minéralisation $\left(\mathrm{R}^{2}+\mathrm{PIR}^{2}\right)$ sur des images satellites Landsat MSS de 1979 et Spot de 1992 (fig. 4; Taïbi et al., 2006). L'ouverture du couvert végétal associé à la salinisation des sols et la dessiccation prolongée des fonds de cuvettes pendant la saison sèche, a favorisé une forte éolisation qui a accentué la dégradation des formations arborées. Les matériaux prélevés par déflation et redéposés par le vent ont formé ainsi des champs de nebkhas notamment dans le bassin du Diawling et encore visible aujourd'hui en rive sénégalaise dans le Parc National des Oiseaux du Djoudj (photo 1).

Une mortalité importante des Tamarix senegalensis a aussi été constatée dans un premier temps, surtout dans les zones les plus basses, même si aujourd'hui cette espèce semble au contraire en phase de colonisation rapide en cours de cartographie.

La retenue du barrage est marquée par la prolifération d'hydrophytes envahissantes, notamment Typha spec., mise en évidence grâce à des classifications des 3 premières composantes d'analyses en composante principale réalisées sur des images satellites masquées de 1992 et 2001 (fig. 5; Taïbi et al., 2006), et Salvinia molesta dont la colonisation a pu être maîtrisée depuis.

La faune halieutique du bas-delta a été également fortement touchée, ce qui s'est traduit par la diminution des captures par les pêcheurs (principalement des espèces estuariennes) accompagnée d'un changement de la nature des espèces (Diawara, 1997). Cette diminution de la ressource halieutique, associée à la disparition des lieux de nidification liée à la dégradation de la couverture végétale, a eu de graves conséquences sur la faune ornithologique avec une forte réduction du nombre d'oiseaux migrateurs comme l'on souligné les comptages d'oiseaux annuels côté mauritanien comme sénégalais réalisés dans les parcs nationaux. Le bas delta (Diawling et Djoudj) accueille en effet d'importantes populations de migrateurs paléarctiques.

Sur le plan socio-économique, l'altération des écosystèmes a entraîné la disparition de la plupart des activités, notamment la cueillette de Sporobolus pour la confection de nattes, la récolte de 


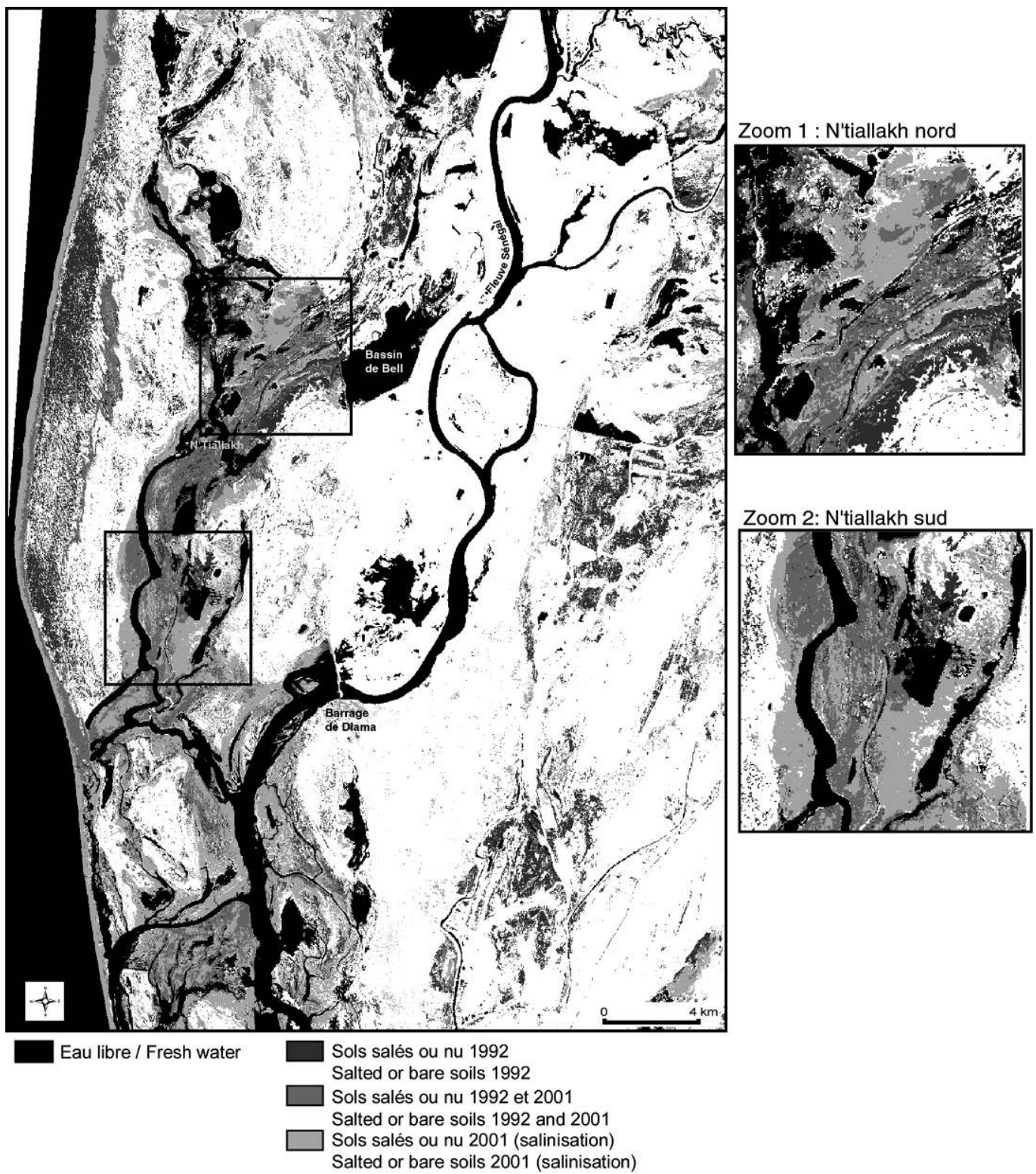

Figure 3 : Sables vifs et processus de salinisation des sols entre 1992 et 2001 (masques à partir de classifications diachroniques non supervisées sur les 3 premiers axes d'ACP des canaux bruts de 1992 et 2001). Bas delta du fleuve Sénégal. (Taïbi et al., 2006)

Sharp sands and soils salinisation from 1992 to 2001 (mask from unsupervised classifications using the 1992 and 2001 raw data 3 first PCA components). River Senegal low delta. (Taïbi et al., 2006)

Nymphea lotus ou des gousses d'Acacia nilotica qui servent pour le tannage des peaux (Duvail, 2001). La pêche, autrefois activité procurant des revenus substantiels pour la population est devenue de plus en plus difficile avec la diminution des captures et à cause des plantes envahissantes. 
Aude Nuscia TAÏBI ET AL.

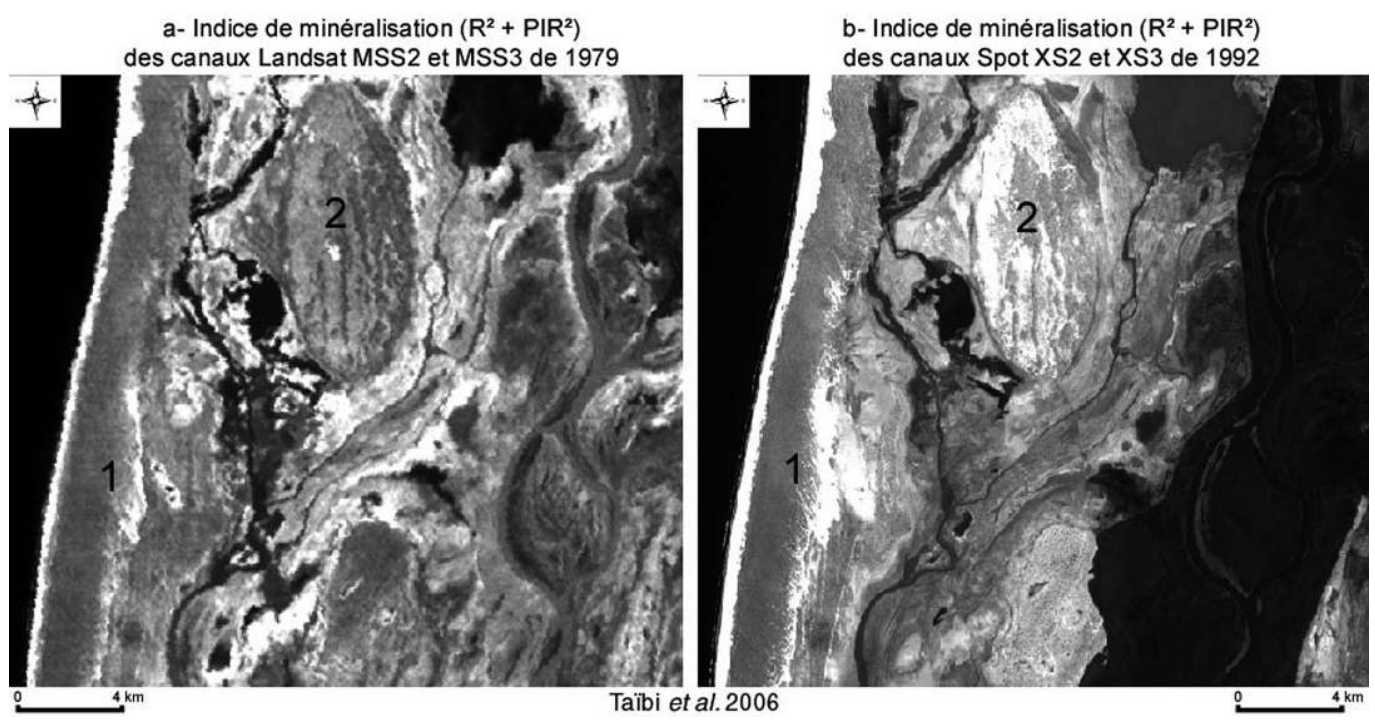

Figure 4 : Réactivation des formations sableuses des crêtes de la dune côtière (1) et de la dune de Ziré (2) (en blanc en 1992) entre 1979 (a) et 1992 (b), Bas delta du fleuve Sénégal. (Taïbi et al., 2006)

Sharpening of coastal dunes crest (1) and Ziré dune (2) (in white in 1992) between 1979 (a) and 1992 (b). River Senegal low delta. (Tä̈bi et al., 2006)

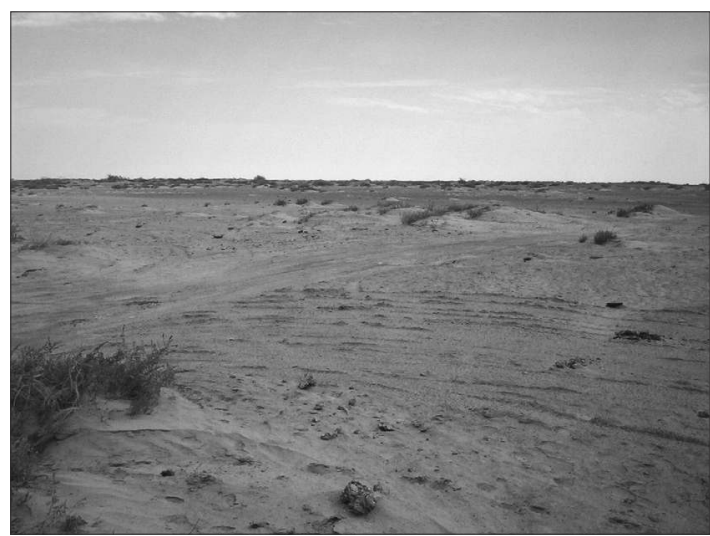

Photo 1 : Déflation et champs de nebkas entre le grand lac et le Gorom, PNOD. (Cliché A. N. Taïbi, fév. 2006)

Deflation and nebkas between the "Grand lac" and the Gorom, PNOD.
Cette période après barrage a également été marquée par une recrudescence de maladies hydriques dans toute la vallée avec un accroissement du nombre de personnes atteintes (paludisme, bilharziose urinaire et intestinale). L'alimentation en eau potable est paradoxalement difficile pour les animaux (pas de couloirs de passage vers le fleuve) et les résidants dans certaines localités et en outre la qualité de l'eau est douteuse. La salinité de l'eau des puits, même ceux situés à proximité du lit du fleuve, n'a cessé d'augmenter.

Du côté sénégalais, les conséquences ont été identiques. Il faut y ajouter le déplacement forcé des populations d'éleveurs peulhs remplacés par des agriculteurs (riz), la prolifération de Pistia stratiotes qui s'ajoute à celle de Typha spec. et des problèmes de pollution des eaux et des sols par les phytosanitaires utilisés dans les périmètres rizicoles

\section{LES IMPACTS DU BARRAGE D’ARZAL SUR LA VILAINE}

Dans l'estuaire de la Vilaine, l'impact sur les milieux à l'aval du barrage est également très fort et ont été également rapidement mis en évidence par les travaux de F. Maillocheau (1980) par exemple. La limitation des échanges amont-aval au niveau de l'estuaire favorise la prédominance des phénomènes hydrodynamiques d'origine marine. En l'absence d'effet de chasse provoqué par les eaux fluviales, notamment en période de hautes eaux, l'estuaire fonctionne alors comme une 


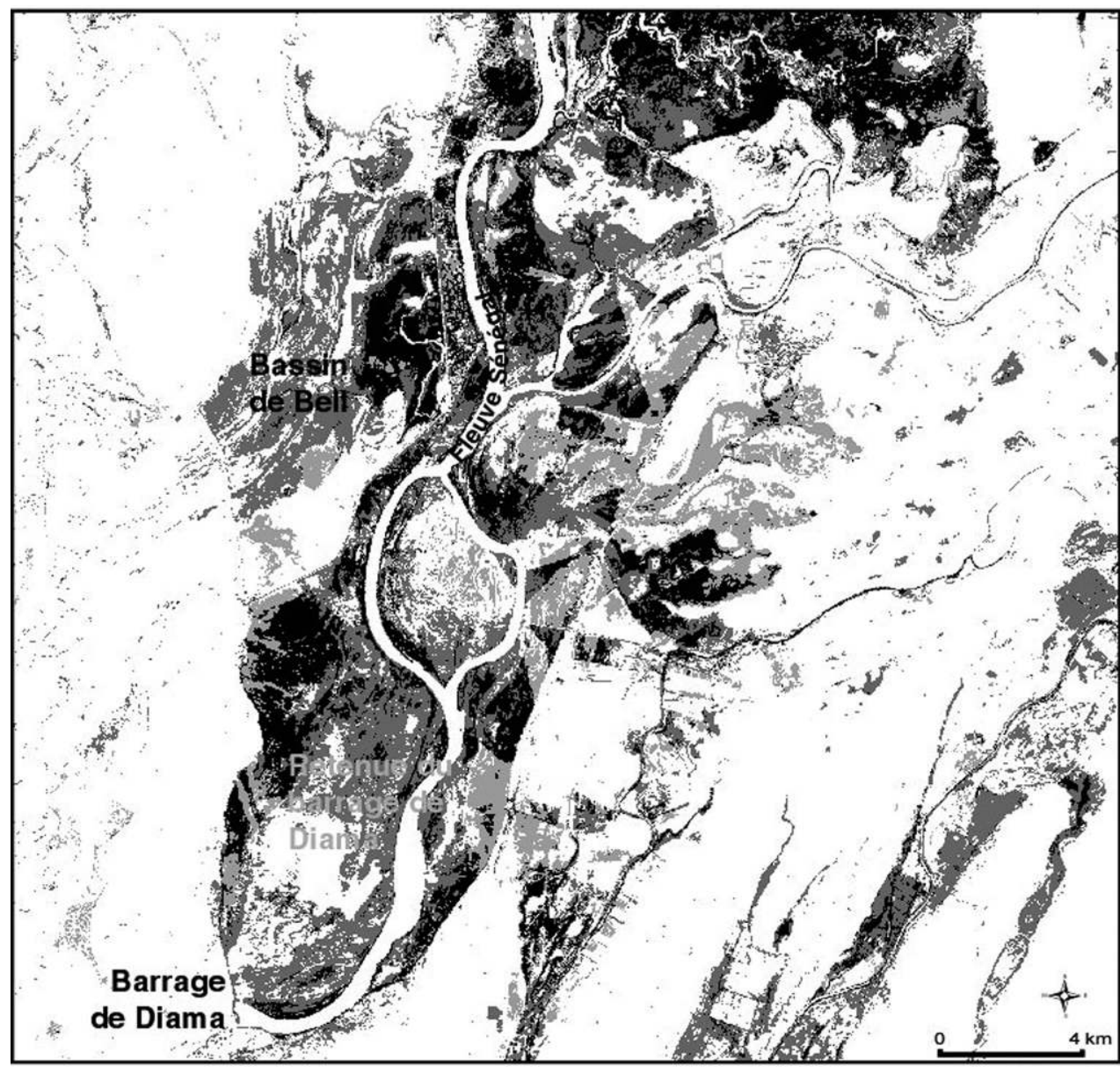

Absence de Typha Missing Typha

Typha dense 1992 / Dense Typha 1992

Proliferation du Typha de 1992 à 2001 (absent en 1992) Typha proliferation from 1992 to 2001 (missing in 1992)

Typha dense 1992 et $2001 /$ Dense Typha 1992 and 2001

Sauf zones hors retenue de Diama / except areas out of Diama dam

Figure 5 : Prolifération de Typha sp. dans le PND (Classification non supervisée diachronique des 3 premiers axes d'ACP des canaux bruts de 1992 et de 2001). (Taïbi et al., 2006)

Typha sp. proliferation in the PND (Unsupervised classification using the 1992 and 2001 raw data 3 first PCA components). (Taïbi et al., 2006)

baie semi-fermée. Ceci se caractérise par une séquence régressive, la taille des particules sédimentées diminuant du large vers le fond de la baie (Allen, 1972; Debenay et al., 1991). L'eau salée venant de l'océan, rythmée par les marées, pénètre dans l'estuaire au ras du fond (l'eau salée est plus lourde que l'eau douce et passe donc au-dessous de celle-ci), apporte des sédiments marins grossiers et les dépose à l'intérieur de l'estuaire. D'autre part, l'eau douce venant du continent et restant davantage en surface, apporte des sédiments plus fins (Pinot, 1998). Toutes les baies connaissent ce phénomène et leur évolution actuelle est donc de se combler (baie du Mont saint Michel, Arcachon, etc.).

Mais cette sédimentation, essentiellement de particules fines, a été ici artificiellement accélérée et l'estuaire s'envase rapidement (fig. 6; Goubert et Menier, 2005). La totalité du volume 


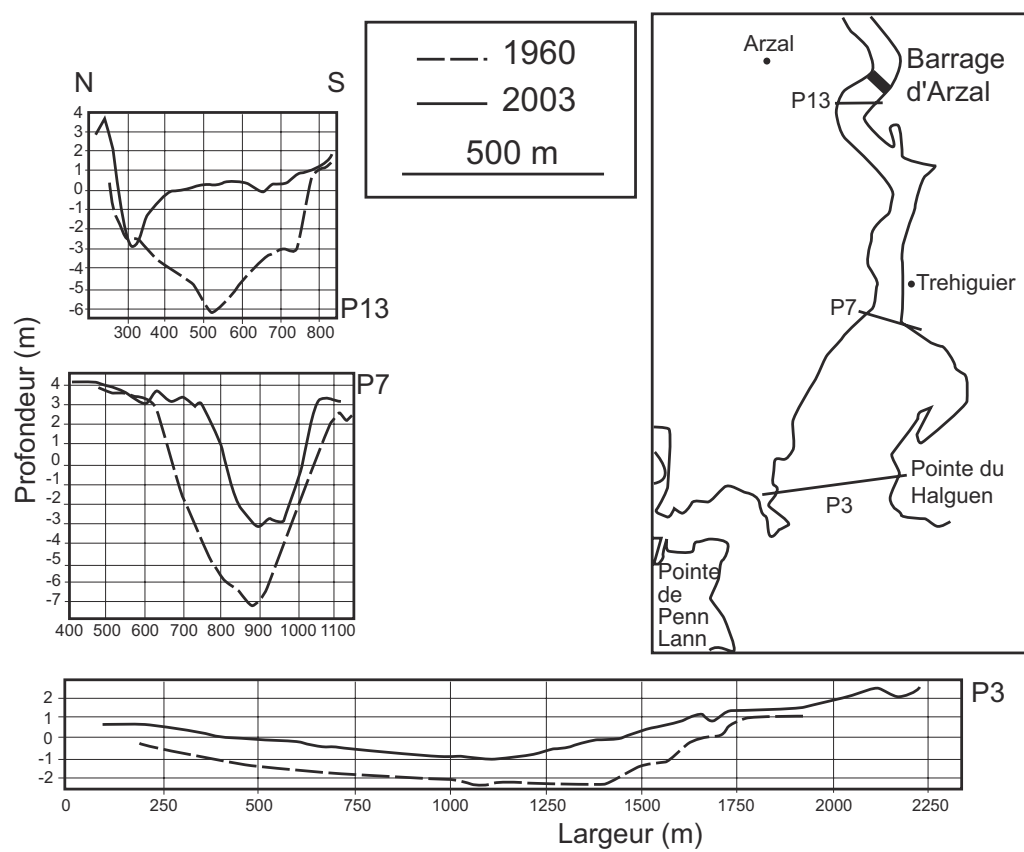

Figure 6 : Évolution de la profondeur du chenal de l'estuaire de la Vilaine de 3 sites entre 1960 et 2003 (Goubert et Menier, 2005).

Vilaine

estuary channel depth evolution in 3 sites between 1960 and 2003 (Goubert et Menier, 2005)

sédimentaire déposé depuis la mise en place du barrage (1970) est d'environ 8 millions de $\mathrm{m}^{3}$ entre le barrage et Tréhiguier, soit $1460 \mathrm{~m}^{3}$ par mètre linéaire et une hauteur totale moyenne de près de 4 mètres en un quart de siècle. Les zones interne et intermédiaire de l'estuaire ont connu une phase de colmatage importante de 1960 à 1994. Plus particulièrement jusqu'en 1983, c'est $80 \%$ du volume total de vase qui s'est déposé dans la zone interne, soit $6380000 \mathrm{~m}^{3}$. De 1983 à 1994, il y a un net ralentissement avec des dépôts trois fois inférieurs à la période précédente. Ce processus s'accompagne d'une méandrisation et d'un rétrécissement du chenal de 350-500 m avant barrage à 80-150 m après (IAV, 1995; Menier et Goubert, 2003). Au-delà de 1994, si certains sites (chenal particulièrement) connaissent un bilan sédimentaire négatif avec une érosion du chenal de navigation lié à des épisodes de crues exceptionnelles (1995 par exemple), le port de Tréhiguier notamment continue à s'envaser. Par ailleurs, il semble que cette relative stabilisation de l'envasement masque ces mêmes types de processus dans la zone externe, difficiles à mesurer.

Si l'extension des vasières en aval d'Arzal a favorisé le développement des populations d'organismes qui occupent les vasières comme les organismes fouisseurs ou les oiseaux, elle a en revanche affecté les peuplements piscicoles qui se sont globalement appauvris depuis les années 1980 en liaison notamment avec la réduction de la longueur de l'estuaire de 40 à 10 km qui a entraîné la réduction des surfaces d'échanges au niveau des vasières, la diminution des courants et l'artificialisation des débits (Desaunay et Guerault, 2003)

Cet envasement a également affecté les exploitations conchylicoles qui ont dû se déplacer et la vue des parcs abandonnés est impressionnante. Le redéploiement des mytiliculteurs vers l'extérieur de l'estuaire a débuté dès 1972 comme à Cromenach par exemple (Le Grel et Philippe, 2003). Aujourd'hui, les concessions ne se localisent plus qu'entre Halguen et Les Granges et des projets de lotissements conchylicoles sont en cours de réalisation à l'extérieur de l'estuaire au sud de la pointe de Halguen (commune de Penestin). Ces difficultés de l'activité mytilicole sont liées non seulement directement à l'envasement des sites de culture mais aussi à l'insuffisance de l'approvisionnement en eau des bassins de stockage en partie envasés ainsi qu'à des problèmes de débarquement qui gênent l'activité. 
Les activités touristiques (plaisanciers, etc.) ont également été touchées, l'envasement des plages étant un élément fortement répulsif, ainsi que la pêche, Arzal formant un obstacle pour la remontée des poissons migrateurs, anguilles, saumons ou aloses. Seule la pêche à la civelle s'est accrue, notamment au niveau de la passe à poisson d'Arzal, dégageant 3,5 millions d'euros de chiffre d'affaire par an (Jolivel, 2005).

\section{Des tentatives de remédiation aux impacts négatifs des barrages}

\section{LE BAS DELTA DU FLEUVE SÉNÉGAL}

Dans le bas delta du fleuve Sénégal, c'est pour tenter de remédier à ces impacts négatifs que le Parc National du Diawling (PND) est officiellement créé en janvier 1991 en Mauritanie, avec le statut d'aire protégée (décret 1991-005). Ses objectifs (Hamerlynck, 1996) sont de restaurer ces espaces dégradés du bas delta et de concilier la protection de l'environnement (« conservation et utilisation durable des ressources naturelles d'un échantillon de l'écosystème du bas-delta ») et le développement socio-économique des collectivités ayant traditionnellement des droits d'exploitation dans la zone ( « développement harmonieux et permanent de diverses activités des populations locales et la coordination des activités menées sur son territoire »). Couvrant 16000 hectares du bas-delta, le PND (56000 hectares avec sa zone périphérique) est basé sur un fonctionnement hydraulique totalement artificiel (fig. 1c). Il comprend trois bassins; Diawling (7500 ha), Bell (4000 ha) et Gambar (4500 ha), alimentés par des ouvrages hydrauliques vannés construits aux points de recoupement de digues avec les principaux marigots, et permettant une alimentation en eau contrôlée de la plaine d'inondation à partir du fleuve par le biais des deux ouvrages de Cheyal et de Lemer (fig. 1c). Pour des raisons d'équilibres écologiques et d'exploitation économique, l'eau ne doit pas être gardée en permanence dans tous les bassins du parc. Ces ouvrages sont ouverts et fermés selon les scénarii de gestion du barrage. L'ouverture est en principe fixée au début du mois de juillet et la fermeture trois mois plus tard. Lorsque les vannes sont ouvertes et la crue suffisante, tous les marigots entrent en communication à travers les nombreuses cuvettes de décantation qu'ils partagent et inondent rapidement (d'amont en aval). La période d'assèchement progressif du parc va en général de janvier à mars notamment grâce à l'ouvrage de Bell $2\left(15 \mathrm{~m}^{3} / \mathrm{s}\right)$ qui permet d'alimenter le bassin du Thiallakht, où l'eau est saumâtre le bassin étant situé en aval du barrage (fig. 1c). L'évaporation contribue également à cet assèchement. Deux autres ouvrages du parc, celui de Bell $1\left(5 \mathrm{~m}^{3} / \mathrm{s}\right)$ et celui de Lekser $\left(5 \mathrm{~m}^{3} / \mathrm{s}\right)$ qui sont souvent laissés ouverts, jouent en conditions optimales un double rôle : à marée haute dans le bassin du Thiallakht, ils permettent de petites incursions d'eau saumâtre dans le parc et, en situation inverse, ils évacuent le trop plein d'eau douce du parc, même si l'ouvrage de Bell 2 est fermé. L'espace du Bas Delta étant compartimenté, les autres ouvrages (Berbare, etc.) assurent le transit inter bassins de l'eau (jusqu'au Chott Boul).

Cette restauration des inondations contrôlées depuis 1994 associées à de meilleures pluies, ont favorisé une bonne régénération de différents groupements végétaux en de nombreux sites, notamment dans les cuvettes et sur les dunes. Les formations herbacées annuelles et pérennes (Sporobolus robustus, Sueda mollis, Echinochloa, etc.) se développent à nouveau après la décrue dans les différents bassins, limitant la déflation éolienne (Hamerlynck et Duvail, 2003). Les formations arborées ont connu également une bonne régénération, notamment Acacia nilotica ou Tamarix senegalensis dans le bassin de Bell. Les palétuviers Avicennia qui avaient fortement régressé avec l'accroissement de la salinité, sont en train de reconquérir l'estuaire (fig. 7 et photos 2 et 3) où l'on rencontre de nombreuses propagules (Gonzalez, 2005).

Cette régénération a favorisé le retour des activités liées à ces écosystèmes et qui avaient disparu avec leur dégradation. Les pêcheurs ont repris la pêche et les femmes la cueillette et l'artisanat (tissage de natte à partir de Sporobolus et Typha et tannage des peaux à partir des gousses d'Acacia nilotica). Les pâturages régénérés sont également de nouveau fréquentés. 


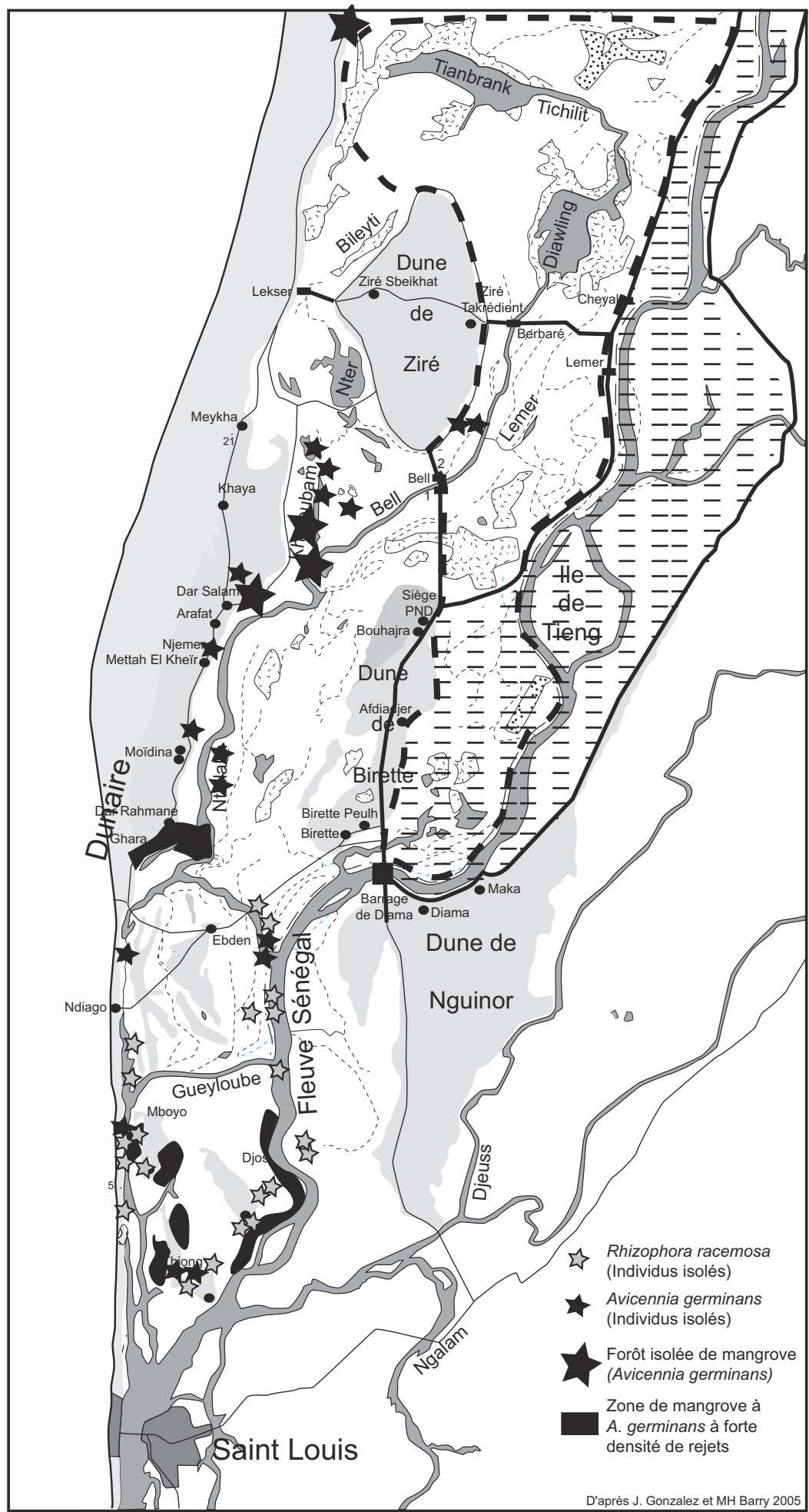

Figure 7 : Répartition de la mangrove dans le bas delta du fleuve Sénégal (2005)

\section{Spatial}

distribution of the mangrove in the river Senegal low delta (2005) 


\section{2}

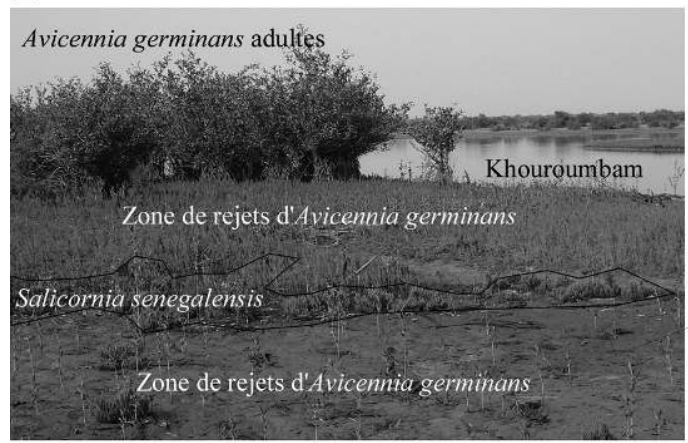

3

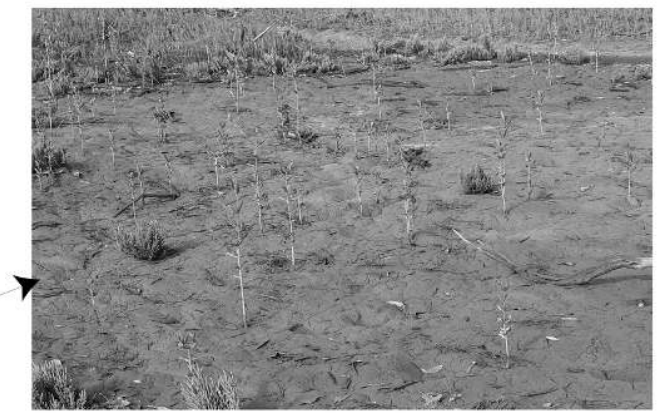

Photo 2 : Mangrove en rive est du Khouroumbam (PND) (Cliché J. Gonzalez, 2005). Mangrove on the east side of Khouroumbam (PND)

Photo 3 : Rejets d'Avicennia germinans en rive est du Khouroumbam (PND) (Cliché J. Gonzalez, 2005) Avicennia germinans tiller on the east side of Khouroumbam (PND)

\section{L'ESTUAIRE DE LA VILAINE}

Dans l'estuaire de la Vilaine, outre le fait qu'une passe à poisson mise en place en 1996 a permis la remontée des migrateurs auparavant bloqués par le barrage, c'est surtout la création d'un SAGE, qui est le plus grand de France, s'étendant sur environ $11000 \mathrm{~km}^{2}$ [http://www.lavilaine.com] et couvrant six départements, qui a permis la mise en place, dans la zone inondable, de pratiques plus adaptées à la fragilité du milieu. Ses objectifs, classiques, sont la restauration de la qualité des eaux brutes, la production d'eau potable, l'amélioration de l'assainissement, la gestion des prélèvements d'eau, la régulation des étiages et des crues et la restauration de la qualité et des fonctions des milieux sensibles. Par ailleurs, des dragages réguliers sont opérés dans l'estuaire de la Vilaine pour lutter contre l'envasement. Trois campagnes ont été réalisées en 1999 et une en 2001 évacuant vers le large respectivement $25000 \mathrm{~m}^{3}$ et $17000 \mathrm{~m}^{3}$ de vase (fig. 1b). L'IAV a récemment investit dans un rotodévaseur pour des actions de dévasement ponctuelles notamment devant l'écluse du barrage au port de Tréhiguier. Elles permettent surtout de faciliter la navigation de plaisance et de pêche [http://wwww.lavilaine.com].

\section{Des mesures compensatoires tardives et aux résultats mitigés}

Mais la plupart de ces mesures compensatoires ont été le plus souvent très tardives dans les deux cas et leurs résultats relativement mitigés.

Ainsi, des travaux récents (Taïbi et al., 2006) montrent bien que si le Parc National du Diawling sur le fleuve Sénégal a permis la restauration des écosystèmes de cette portion du bas delta, il n’a pas pour autant résolu tous les problèmes de dégradation de ces milieux.

Le compartimentage de l'espace par les digues et le barrage et l'inondation, a fortement réduit l'espace des activités humaines pendant cette période, alors même qu'elle est la seule à être propice à ces mêmes activités de pêche, de cueillette, de commerce (écoulement des produits) ou d'élevage. Par ailleurs, les pressions exercées sur cet espace restreint n'ont cessé de s'accroître en raison de la forte attractivité nouvelle liée à cette régénération et à « l'abondance » du moment, générant de nouveaux risques de dégradation et d'usage.

Dans l'estuaire de la Vilaine les dragages opérés régulièrement pour lutter contre l'excès de sédimentation ne sont pas non plus sans conséquences notamment sur les organismes qui occupent les fonds marins au large qui accueillent les sédiments dragués. Par ailleurs, ces dragages ont un coût élevé et créent également des conflits d'usage comme lors du dragage du port de Tréhiguier 
Aude Nuscia TAÏBI ET AL.

en 1998 qui a généré une accumulation de sédiments sur les plages de la rive droite, mécontentant fortement les plagistes et l'association de défense et de sauvegarde de la baie de Vilaine.

Il est intéressant de noter qu'une enquête auprès de producteurs (Jolivel, 2005) met en évidence leur scepticisme vis-à-vis de l'efficacité des mesures de remédiation par rapport à leur activité. En effet, interrogés sur les préjudices de l'envasement sur leurs activités, $57 \%$ les jugent ingérables (29\% très forts et 14 \% forts).

L'illusoire maîtrise des inondations par le barrage d'Arzal a favorisé l'occupation de la zone inondable par des aménagements industriels et commerciaux dans la région de Redon accroissant ainsi l'impact des crues que l'on voulait diminuer.

Le développement de l'activité de plaisance a également eu des effets induits insoupçonnés, notamment en période estivale, sur la qualité des eaux de la retenue dont la salinité s'accroît beaucoup avec les nombreuses écluses permettant le passage de bateaux de plaisance en période d'étiage. Cela impose la fermeture parfois pendant plusieurs jours de l'écluse pour rétablir la qualité de l'eau.

\section{Conclusion}

Que ce soit dans les pays développés ou en voie de développement, on constate que lorsque des aménagements sont stratégiques, et c'est le cas lorsque l'on a besoin de développer la ressource en eau dans des pays où cette ressource est insuffisante, ceci quelles que soient les conditions climatiques, les impacts potentiels sur l'environnement et les activités, notamment traditionnelles, ne constituent pas une priorité pour les décideurs face aux contraintes économiques. Dans le cas de ces deux barrages d'Arzal et de Diama, aucune quantification des coûts/bénéfices n'a été réalisée, pourtant les instabilités sociales et économiques voire politiques (événements sénégalomauritaniens en 1989) générées font se poser la question de l'intérêt de ce type de grands ouvrages aux conséquences difficilement maîtrisables.

On peut comprendre aisément cette prééminence des objectifs économiques de court terme, mais cela devrait s'accompagner de mesures compensatoires efficaces, qui permettent de diminuer les impacts sur les milieux et activités. Ces mesures apparaissent progressivement par nécessité mais trop souvent très tardivement; près de 10 ans pour que le Parc National du Diawling et son système hydraulique artificialisé soit opérationnel, ou 26 ans pour la passe à poisson d'Arzal par exemple.

En tout état de cause, la présence de ces ouvrages aussi stratégiques et aussi nécessaires soientils, et les mesures compensatoires qui les accompagnent, comme les expériences du Parc National du Diawling en Mauritanie et du SAGE Vilaine en France, ne permettront pas de retrouver l'état initial des milieux. Ces louables et importants efforts de remédiation des conséquences environnementales et socio-économiques des barrages, montrent que la restauration des écosystèmes et des activités n'est jamais chose aisée.

Les objectifs de 2015 de la Directive Cadre Européenne auront du mal à être atteints dans l'estuaire de la Vilaine, comme à l'amont proche. Mais qui pourrait imaginer que l'on puisse effacer Arzal et supprimer la ressource en eau de 1,5 million d'habitants?

Dans le bas delta du fleuve Sénégal, de nouveaux efforts de remédiation à échelle plus large sont réalisés avec la création de la Réserve de Biosphère Transfrontalière (RBT) en 2000 couvrant les deux rives du fleuve Sénégal et prenant donc en compte le caractère systémique du fonctionnement de ces zones humides. Mais le démarrage effectif est lent et il subsiste toujours la principale difficulté des enjeux et objectifs différents entre institutions d'aménagement et gestion du fleuve (OMVS) et Parcs et réserves (RBT, PND et Parc National des Oiseaux du Djoudj au Sénégal). 


\section{Bibliographie}

AlLEN (G.-P.), 1972. - Étude des processus sédimentaires dans l'estuaire de la Gironde, Thèse de doctorat en Sciences Naturelles, Université de Bordeaux I, 314 p.

BarRy (M. H.), 2004. - Le Parc National du Diawling dans le processus de développement durable du Bas Delta mauritanien du fleuve Sénégal : conflits d'usages et d'acteurs dans un contexte de changements environnementaux et socio-économiques, Mémoire de DEA, Université d'Angers, 199 p.

BDPA/SCETAGRI/ORSTOM/SECA/AFID/SERADE, 1994. - Étude des problèmes d'environnement et de protection des milieux naturels dans le delta du fleuve Sénégal (5 tomes), Tome 1, Phase I, Bilan et diagnostic, OMVS/FAC, Dakar, 55 p.

Debenay (J.-P.), Guillou (J.-J.), Pages (J.), Ba (M.), Moguedet (G.), Perthuisot (J.-F.), Ponthoreau-Granet (C.), 1991. - L'écosystème de la Casamance en mai 1990. Évolution de 1984 à 1990. Étude des estuaires du Sénégal: Sénégal, Saloum, Casamance, Dakar, UNESCO/BREDA/PNUD/EPEEC, Rapport final, 99 p.

DÉSAUnAY (Y.), GUÉRAULT (D.), 2003. - Évolution du peuplement halieutique de la baie de Vilaine au cours des décennies 1980 et 1990, IFREMER/DRV/RH/ECOHAL , 24 p. [www.ifremer.fr/docelec/doc/2003/rapport-1288.pdf].

DiaWARA (Y.), 1997. - « Formations morphopédologiques et unités floristiques du bas delta mauritanien », dans Colas (F.), Environnement et littoral mauritanien, Actes du colloque de juin 1995, Nouakchott (Mauritanie), Montpellier, CIRAD, p. 47-52.

Duvail (S.), 2001. - Scénarios hydrologiques et modèle de développement en aval d'un grand barrage. Les usages de l'eau et le partage des ressources dans le delta mauritanien du fleuve Sénégal, Thèse de doctorat de géographie, Université Louis Pasteur Strasbourg I, 313 p.

GonZalez (J.), 2005. - Plan de gestion pour la régénération de la mangrove dans le parc National du Diawling et sa périphérie (Mauritanie), Rapport de stage DESS «Gestion des Zones Humides. Biodiversité et ingénierie », Université d'Angers, 60 p. et annexes.

Goubert (E.), Menier (D.), 2005. - Évolution morphosédimentologique de l'estuaire de la Vilaine de 1960 à 2003 : valorisation des campagnes bathymétriques, Mission d'assistance à la tenue et à la valorisation des données bathymétriques de l'estuaire de la Vilaine, IAV, Université de Bretagne Sud, 20 p. et annexes.

Hamerlynck (O.), 1996. - Plan de gestion du Parc National du Diawling et de sa zone périphérique 1996-2001, Mauritanie, PND, UICN, 63 p.

Hamerlynck (O.), Duvail (S.), 2003. - La restauration du delta du fleuve Sénégal en Mauritanie, Gland (Suisse)/Cambridge (Royaume-Uni), UICN, coll. «Série bleue VIII », 88 p.

Institut d’Aménagement de la Vilaine (IAV), 1995. - Étude de l'envasement de l'estuaire de la Vilaine, rapport final ERAMM-RIVAGES, déc. 1995, p. 106 [http://www.lavilaine.com].

Jolivel (M.), 2005. - Envasement et dragage de l'estuaire de la Vilaine : à la recherche de solutions durables par un consensus entre les acteurs, Mémoire de maîtrise, Université d'Angers, 113 p.

Le Grel (L.), Philippe (M.), 2003. - Étude d'évaluation du développement de la conchyliculture de l'estuaire de la Vilaine, Ö̈kos Environnement-Ressources, Rapport final, 58 p. [http://www.lavilaine.com].

Maillocheau (F.), 1980. - L'envasement de l'estuaire de la Vilaine en aval du barrage d'Arzal, Mémoire de DEA, Université de Nantes, 65 p.

Menier (D.), Goubert (E.), 2003. - Mission d'assistance à la tenue et à la valorisation des données bathymétriques de l'estuaire de la Vilaine, 23 p. [http://www.lavilaine.com].

Michel (P.), BARusseau (J.-P.), RichaRd (J.-F.), SALL (M.), 1993. - L'après-barrages dans la vallée du Sénégal; modifications hydrodynamiques et sédimentologiques. Conséquences sur le milieu et les aménagements hydro-agricoles, Paris/Perpignan, Ministère de la Coopération et du Développement/Presses Universitaire de Perpignan, 152 p.

PinOt (J.-P.), 1998. - La gestion du littoral, Tome II : Littoraux tempérés : littoraux vaseux et embouchures, Paris, Institut océanographique, coll. «Propos », 759 p.

Taïbi (A. N.), Barry (M. H.), Hallopé (A.), Moguedet (G.), Ballouche (A.), Ould Baba (M. L.), Ba (A.), 2006. - « Diagnostic par télédétection satellitaire des impacts environnementaux et socio-économiques du Parc National du Diawling sur le Bas Delta du fleuve Sénégal », dans Symoens (J.-J.), Les Écosystèmes côtiers de l'Afrique de l'Ouest. Diversité biologique - Ressources - Conservation, Bruxelles, FFRSA, CNBSB, PRCZCMAO, p 211-229. 
Aude Nuscia TAÏBI ET AL.

\section{Remerciements}

Les images satellites SPOT du bas delta du PND traitées dans cette étude ont été acquises dans le cadre du programme ISIS du CNES (dossier n 0207-350).

Les études sur le PND sont financées par l'AUF dans le cadre d'une Action de recherche en réseau " télédétection et développement durable" n P2-2092RR515 et un Projet partagé de recherche PCSIU «Développement et environnement » $n^{\circ} 6313$ PS586 associant les Universités d'Angers et Caen (France), Nouakchott (Mauritanie), Cheikh Anta Diop de Dakar et Gaston Berger de Saint Louis (Sénégal).

Nous remercions D. Menier (Université de Bretagne Sud) et S. Suanez pour leurs remarques qui ont permis d'améliorer l'article.

Cet article a été reçu le 18 janvier 2007 et définitivement accepté le 22 juin 2007. 